Chapter 5 LIST OF REFERENCES 
This page is intentionally left blank 


\section{References *}

Abbott D.C., 1978, "The Terminal Velocity of Stellar Winds from Early-Type Stars", Astrophys. J., 225, 893. [29].

Abramowicz M.A. and Lasota J.P., 1980, "Spin-up Black Holes by Thick Accretion Disks", Acta Astr., 30, 35. [27]

Abramowicz M.A. and Marsi C., 1987, "Accretion Research: How It Started", Observatory, 107, 245. [1]

Abramowicz M.A. and Piran T., 1980, "On Collimation of Relativistic Jets from Quasars", Astrophys. J. Lett., 241, L7. [24, 27]

Abramowicz M.A., Calvani M. and Nobili L., 1980, "Thick Accretion Disks with SuperEddington Luminosities", Astrophys. J., 242, 772. [1, 27]

Abramowicz M.A., Jaroszynski M. and Sikora M., 1978, "Relativistic, Accreting Disks", Astron. Astrophys., 63, 221. [24, 25, 27]

Abramowicz M.A., Czerny B., Lasota J.P. and Szuszkiewicz E., 1988, "Slim Accretion Disks", Astrophys. J., 332, 646. [1]

Adams W.S. and Joy A.H., 1922, Pop. Astr., 30, 103. [6]

Agrawal P.C., Biswas S., Gokhale G.S., Iyengar V.S., Kunte P..K., Manchanda R.K. and Sreekantan B.V., 1970, "Energy Spectra of Several Discrete X-Ray Sources in the 20-120 keV Range", in Non-solar X-and $\gamma$-Ray Astronomy, I.A.U. Symposium No. 37, ed. L.Gratton (Dordrecht: Reidel). [18]

Aizu K., 1973, "X-Ray Emission Region of a White Dwarf with Accretion", Progr. Theor. Phys., 49, 1184. [1, 18, 21]

Allen C.W., 1973, Astrophysical Quantities, 3rd edition, Athlone Press, London. [21]

Alme M.L. and Wilson J.R., 1973, "X-Ray Emission from a Neutron Star Accreting Material", Astrophys. J., 186, 1015. [1, 16, 18]

Ambartsumjan V.A. and Saakyan G.S., 1960, "The Degenerate Superdense Gas of Elementary Particle", Astron. Zh., 37, 193 [Sov. Astron.-AJ, 4, 187]. [9]

* This list contains references reported in the 29 papers of this collection. In parenthesis we give the number of the paper which refers to the article. 
Ambartsumjan V.A. and Saakyan G.S., 1963, Voprosy Kosmog., 9, 91. [9]

Amnuehl' P.R. and Gusejnov O.Kh., 1968, Izv. Akad. Nauk Azerbaidzhan SSSR, Ser. Fiz. Tekh. Mat., 3, 70. [12]

Amnuehl' P.R. and Gusejnov O.Kh., 1976, "On the Lifetime of Bright X-Ray Sources", Astroph. Space Sci., 45, 283. [29]

Angel J.R.P, 1969, "X-Ray Line Emission from Sco X-1", Nature, 224, 160. [13]

Angel J.R.P and Stockman H.S., 1980, "Optical and Infrared Polarization of Active Extragalactic Objects", Ann. Rev. A. A., 18, 321. [27]

Arnett W.D., 1966, "Gravitational Collapse and Weak Interactions", Canad. J. Phys., 44, 2553. [9]

Arnett W.D., 1967, "Mass Dependence in Gravitational Collapse of Stellar Cores", Canad. J. Phys., 45, 1621. [9]

Arons J. and Lea S.M., 1976, "Accretion onto Magnetized Neutron Stars: Structure and Interchange Instability of a Model Magnetosphere", Astrophys. J., 207, 914. [21, 29]

Arp H.C., 1961, "U-B and B-V Colors of Black Bodies", Astrophys. J., 133, 874. [6]

Avni Y., 1978, "Masses of Compact X-Ray Sources", talk presented at 16th General Assembly of the I.A.U., in Highlights of Astronomy, Vol. 4. [29]

Baan W.A. and Treves A., 1973, "On the Pulsation of X-Ray Sources", Astron. Astrophys., 22, 421. [1]

Bahcall J.N. and Wolf R.A., 1977, "The Star Distribution around a Massive Black Hole in a Globular Cluster. II. Unequal Star Masses", Astrophys. J., 216, 883. [28]

Bahcall J.N., 1978, "Masses of Neutron Stars and Black Holes in X-Ray Binaries", Ann. Rev. A. A., 16, 241. [29]

Bahcall J.N. and Chester T.J., 1977, "On the Mass Determination of Hercules X-1", Astrophys. J. Lett., 215, L21. [29]

Bardeen J.M., 1970, "Kerr Metric Black Holes", Nature, 226, 64. [13]

Bardeen J.M., 1973, "Timelike and Null Geodesic in the Kerr Metric", in Black Holes, Les Houches, ed. C. DeWitt and B. DeWitt (New York: Gordon and Breach). $[26]$ 
Bardeen J.M. and Petterson J.A., 1975, "The Lense-Thirring Effect and Accretion Disks around Kerr Black Holes", Astrophys. J. Lett., 195, L65. [27]

Bardeen J.M., Press W.H. and Teukolsky S.A., 1972, "Rotating Black Holes: Locally Nonrotating Frames, Energy Extraction, and Scalar Synchrotron Radiation", Astrophys. J., 178, 347. [15]

Barr P., Pollard G., Sanford P.W., Ives J.C., Ward M., Hine R.G., Longaire M.S., Penston M.V., Boksenberg A., Lloyd C., 1980, "The Variability of 3C 390.3", Mon. Not. R. astr. Soc., 193, 549. [27]

Barr P., 1986, "Rapid X-Ray Variability in Radio-Quiet AGN: A Probe of the Innermost Regions of the Active Nucleus", in The Physics of Accretion onto Compact Objects, ed. K.O. Mason, M.G. Watson and N.E. White (Berlin: Springer). [1]

Basko M.A. and Sunyaev R.A., 1973, "Interaction of the X-Ray Source Radiation. with the Atmosphere of the Normal Star in Close Binary Systems", Astroph. Space Sci., 23, 71. [14]

Bath G.T., 1969, "Dynamical Instabilities in Semidetached Close Binary System with Possible Applications to Novae and Novalike Variables", Astrophys. J., 158, 571. [11]

Bath G.T., 1972, "Time-dependent Studies of Dynamical Instabilities in Semidetached Binary Systems", Astrophys. J., 173, 121. [11]

Bath G.T., 1973, "Periodicities and Disks in Dwarf Novae", Nature Phys. Sci., 246, 84. $[11]$

Bath G.T., 1975, "An Oblique Rotator Model for HD Herculis", Mon. Not. R. astr. Soc., 171, 311. [22]

Bath G.T. and Pringle J.E., 1981, "The Evolution of Viscous Discs. I. Mass Transfer Variations", Mon. Not. R. astr. Soc., 194, 967. [1, 22]

Bath G.T. and Pringle J.E., 1982, "The Evolution of Viscous Discs. II. Viscous Variations", Mon. Not. R. astr. Soc., 199, 267. [1]

Bath G.T., Evans W.D. and Pringle J.E., 1974, "Dynamical Instabilities and Mass Exchange in Binary Systems", Mon. Not. R. astr. Soc., 166, 113. [21]

Bath G.T., Evans W.D., Papaloizou J. and Pringle J.E., 1974, "The Accretion Model of Dwarf Novae with Application to Z Chamaleontis", Mon. Not. R. astr. Soc., 
169, 447. [21]

Baym G. and Pethick C.J., 1975, "Neutron Stars", Ann. Rev. Nucl. Sci., 25, 27. [29]

Baym G. and Pethick C.J., 1979, "Physics of Neutron Stars", Ann. Rev. A. A., 17, 415. [29]

Baym G., Pethick C.J., Pines D. and Ruderman M., 1969, "Spin Up in Neutron Stars: the Future in the Vela Pulsar", Nature, 224, 872. [29]

Becker R.H., Rothschild R.E., Boldt E.A., Holt S.S., Pravdo S.H., Serlemitsos P.J. and Swank J.H., 1978, "Extended Observations of Vela X-1 by OSO", Astrophys. J., 221, 912. [29]

Begelman M.C., 1977, "Nearly Collisionless Spherical Accretion", Mon. Not. R. astr. Soc., 181, 347. [28]

Begelman M.C., 1978, "Black Holes in Radiation-dominated Gas: an Analogue of the Bondi Accretion Problem", Mon. Not. R. astr. Soc., 184, 53. [1]

Begelman M.C., 1979, "Can a Spherically Accreting Black Hole Radiate very near the Eddington Limit?", Mon. Not. R. astr. Soc., 187, 237. [1]

Begelman M.C. and Chuieh T., 1988, "Thermal Coupling of Ions and Electrons by Collective Effects in Two-Temperature Accretion Flows", Astrophys. J., 332, 872. [1]

Begelman M.C., Blandford R.D. and Rees M.J., 1984, "Theory of Extragalactic Radio Sources", Rev. Mod. Phys., 56, 255. [1]

Bekefi G., 1966, Radiation Processes in Plasmas (New York: Wiley). [20]

Bisnovatyi-Kogan G.S., 1966, "The Critical Mass of a Hot Isothermal White Dwarf with General Relativistic Effects Taken into Account", Astron. Zh., 43, 89 [Sov. Astron.-AJ, 10, 69 (1966)]. [9]

Bisnovatyi-Kogan G.S., 1979, "Magnetohydrodynamical Processes near Compact Objects", Riv. Nuovo Cimento, 2, 1. [1]

Bisnovatyi-Kogan G.S. and Blinnikov S.I., 1977, "Disk Accretion onto a Black Hole at Subcritical Luminosity", Astron. Astrophys., 59, 111. [24, 25]

Bisnovatyi-Kogan G.S. and Fridman A.M., 1969, "A Mechanism for Emission of X-Rays by a Neutron Star", Astron. Zh., 46, 721 [Sov. Astron.-AJ, 13, 566 (1970)]. [12, 16] 
Bisnovatyi-Kogan G.S. and Sunyaev R.A., 1971, "Galaxy Nuclei and Quasars as Infrared Emission Sources", Astron. Zh., 48, 881 [Sov. Astron.-AJ, 15, 697 (1972)]. [14, 16]

Blaes O. M., 1987, "Stabilization of Non-axisymmetric Instabilities in a Rotating Flow by Accretion on to a Central Black Hole", Mon. Not. R. astr. Soc., 227, 975. [1]

Blandford R.D., 1976, "Accretion Disk Electrodynamics. A Model for Double Radio Sources", Mon. Not. R. astr. Soc., 176, 465. [27]

Blandford R.D. and Ostriker J.P., 1978, "Particle Acceleration by Astrophysical Shocks", Astrophys. J. Lett., 221, L29. [28]

Blandford R.D. and Payne D., "Compton Scattering in a Converging Fluid Flow. I. The Transfer Equation", Mon. Not. R. astr. Soc., 194, 1033. [27]

Blandford R.D. and Payne D., "Compton Scattering in a Converging Fluid Flow. III. Spherical Supercritical Accretion", Mon. Not. R. astr. Soc., 196, 781. [27]

Blandford R.D. and Thorne K.S., 1979, "Black Hole Astrophysics", in General Relativity. An Einstein Centenary Survey, ed. S.W. Hawking and W. Israel (Cambridge: Cambridge University Press). [24]

Blandford R.D. and Znajek R., 1977, "Electromagnetic Extraction of Energy from Kerr Black Holes", Mon. Not. R. astr. Soc., 179, 433. [27]

Blumenthal G.R., 1974, "The Poynting-Robertson Effect and Eddington Limit for Electron Scattering with Hard Photons", Astrophys. J., 188, 121. [18]

Blumenthal G.R. and Gould R.J., 1970, "Bremsstrahlung, Synchrotron Radiation, and Compton Scattering of High-Energy Electrons Traversing Dilute Giases", Rev. Mod. Phys., 42, 237. [18]

Bohm D., 1949, The Characteristic of Electrical Discharges in Magnetic Fields, ed. A. Guthrie and R.K. Wakerling (New York: McGraw-Hill). [16]

Bolton C.T., 1972, "Identification of Cygnus X-1 with HDE 226868", Nature, 235, 271. $[13]$

Bondi H., 1952, "On Spherical Symmetrical Accretion", Mon. Not. R. astr. Soc., 112, 195. $[1,4,12,13,17,24]$

Bondi H., 1960, Cosmology (Cambridge: Cambridge University Press). [24] 
Bondi H. and Hoyle F., 1944, "On the Mechanism of Accretion By Stars", Mon. Not. R. astr. Soc., 104, 273. $[3,4,12,16]$

Boynton P.E., Groth E.J., Hutchinson D.P., Nanos G.P., Partridge R.B. and Wilkinson D.T., 1972, "Optical Timing of the Crab Pulsar NP 0532", Astrophys. J., 175, 217. [16]

Bradt H.V. and McClintock J.E., 1983, "The Optical Counterparts of Compact Galactic X-Ray Sources", Ann. Rev. A. A., 21, 13. [1]

Brandt J.C. and Rosen R.G., 1969, "Messier 87: The Galaxy of Greatest Known Mass", Astrophys.- J. Lett., 156, L59. [10]

Bregman J., Butler D., Kemper E., Koski A., Kraft R. and Stone R.P.S., 1973, "On the Distance to Cygnus X-1 (HDE 226868)", Astrophys. J. Lett., 185, L117. [26]

Brown R.L. and Gould R.J., 1970, "Interstellar Absorption of Cosmic X-Ray", Phys Rev. D., 1, 2252. [21]

Buff J.M. and McCray R., 1974, "Accretion Flows in Galactic X-Ray Sources. I. Optically Thin Spherically Symmetric Model", Astrophys. J., 189, 147. [21]

Burbridge E.M., 1967, "Theoretical Ideas Concerning X-Ray Sources", in Radio Astronomy and Galactic System, I.A.U. Symposium No. 31, (London and New York: Academic Press). [8]

Burbridge E.M., Lynds C.R. and Stockton A.N., 1967, "On the Binary Nature of Cyg X-2", Astrophys. J. Lett., 150, L95. [8, 12]

Burger H.L. and Katz J.I., 1983, "The Eddington Limit and Supercritical Accretion. II. Time-dependent Calculations", Astrophys. J., 265, 393. [1]

Byram E.T., Chubb T.A. and Freidman H., 1966, "Cosmic X-Ray Sources, Galactic and Extragalactic", Science, 152, 66. [10]

Callahan P.S., 1977, "On the Accretion Disk Model of QSOs", Astron. Astrophys., 59, 127. $[25]$

Cameron A.G.W. and Mock M., 1967, "Stellar Accretion and X-Ray Emission", Nature, 215, 464. $[8,9,12]$

Canuto V. and Chiu H.Y., 1971, "Intense Magnetic Fields in Astrophysics", Space Sci. Rev., 12, 3. [16] 
Carter B., Gibbons G.W., Lin D.N.C. and Perry M.J., 1976, "Black Hole Emission Process in the High Energy Limit", Astron. Astrophys., 52, 427. [24]

Cassinelli J.P. and Castor J.I., 1973, "Optically Thin Stellar Winds in Early-Type Stars", Astrophys. J., 179, 189. [18]

Castor J.L., Abbott D.C. and Klein R.I., 1975, "Radiation-driven Winds in Of Stars", Astrophys. J., 195, 157. [29]

Chan K.L. and Henriksen R.N., 1980, "On the Supersonic Dynamics of Magnetized Jets of Thermal Gas in Radio Galaxies", Astrophys. J. Lett., 241, 534. [27]

Chandrasekhar S., 1950, Radiative Transfer (Oxford: Oxford University Press). [26]

Chau Wai-Yin, 1967, "Gravitational Radiation from Neutron Stars", Astrophys. J., 147, 664. [9]

Charles P.A., Mason K.O., White N.E., Culhane J.L., Sanford P.W. and Moffat A.F.J., 1978, "X-Ray and Optical Observations of 3U0090-40 (Vela X-1)", Mon. Not. R. astr. Soc., 183, 813. [29]

Chiu H.Y., 1964, "Supernovae, Neutrinos and Neutron Stars", Ann. Phys., 26, 364. [9]

Cline Y.L., Desai U.D., Klebesadel R.W. and Strong I.B., 1973, "Energy Spectra of Cosmic Gamma-Ray Bursts", Astrophys. J. Lett., 185, L1. [18]

Cohen M.H. and Readhead A.C.S., 1979, "Misalignment in the Radio Jets of NGC 6251", Astrophys. J. Lett., 233, L101. [27]

Colpi M., 1988, "Multiple Compton Scattering by Thermal Electrons in a Spherical Inflow: the Effects of Bulk Motion", Astrophys. J., 326, 223. [1]

Colpi M., Maraschi L. and Treves A., 1984, "Two-Temperature Model of Spherical Accretion onto a Black Hole", Astrophys. J., 280, 319. [1]

Colpi M., Maraschi L. and Treves A., 1986, "Gamma-Ray Emission from Accretion onto a Rotating Black Hole", Astrophys. J., 311, 150. [1]

Cominski L., Clark G.W., Li F., Mayer W. and Rappaport S., 1978, "Discovery of 3.6-s X-Ray Pulsations from 4 U 0115+63", Nature, 273, 367. [29]

Condon J.J., Ladder J.E., O'Dell S.L. and Dennison B., 1979, "318-Mhz Variability of Complete Samples of Extragalactic Radio Sources", Astron. J., 84, 1. [27]

Condon J.J., Condon M.A., Jauncey D.L., Smith M.G., Turtle A.J. and Wright A.E., 1981, "A Multifrequency Radio Observations of Optically Selected Quasars", 
Astrophys. J., 244, 5. [27]

Conti P.S., 1978, "Mass Loss in Early-Type Stars", Ann. Rev. A. A., 16, 371. [29]

Cooper J., 1971, "Compton Fokker-Planck Equation for Hot Plasmas", Phys. Rev. D., 3, 2312. [26]

Costero R. and Osterbrok D.E., 1977, "The Optical Spectra of Narrow-Line Radio Galaxies", Astrophys. J., 211, 675. [27]

Cotton W.D., Shapiro I.I. and Wittels J.J., 1981, "Observations of the Jet near the Core of M87", Astrophys. J. Lett., 244, L57. [27]

Cowie L.L., Ostriker J.P. and Stark A.A., 1978, "Time-dependent Spherically Symmetric Accretion onto Compact X-Ray Sources", Astrophys. J., 226, 1041. $[1,28]$

Cowley A.P., 1977, "Low-Mass X-Ray Binaries and their Relation to the Non-X-Ray Sources", Proc. 8th Texas Symposium on Relativistic Astrophysics (Ann. NY Acad. Sci., 302, 1). [29]

Cox D.P. and Tucker W.H., 1969, "Ionization Equilibrium and Radiative Cooling of a Low-Density Plasma", Astrophys. J., 157, 1157. [13, 17]

Crawford J.A. and Kraft R.P., 1956, "An Interpretation of AN Aquarii", Astrophys. J., 123, 44. [11]

Cunningham C., 1975, "The Effects of Redshifts and Focusing on the Spectrum of an Accretion Disk around a Black Hole", Astrophys. J., 202, 788. $[25,26]$

Cunningham C., 1976, "Returning Radiation in Accretion Disks around Black Holes", Astrophys. J., 208, 534. [25]

Czerny B. and Elvis M., 1987, "Constraints on Quasar Accretion Disks from the Optical/ Ultraviolet/Soft X-Ray Big Bump", Astrophys. J., 321, 305. [1]

Dahlbacka G.H., Chapline G.F. and Weaver T.A., 1974, "Gamma Rays from Black Holes", Nature, 250, 36. [20, 26]

Davidsen A., Henry J.P., Middleditch J. and Smith H.E., 1972, "Identification of the X-Ray Pulsar in Hercules: A New Optical Pulsar", Astrophys. J. Lett., 177, L97. [16]

Davidson K., 1973, "Model for Sources Like Cen X-3 and Her X-2", Nature Phys. Sci., 246, 1. [18] 
Davidson K. and Ostriker J.P., 1973, "Neutron-Star Accretion in a Stellar Wind: Model for a Pulsed X-Ray Source", Astrophys. J., 179, 585. [1, 11, 16, 18, 21, 29]

Davies R., Fabian A. and Pringle J., 1979, "Spindown of Neutron Stars in Close Binary Systems", Mon. Not. R. astr. Soc., 186, 779. [29]

Dean A., Gerardi G., De Martinis C., Monastero G.F., Russo A. and Scarsi L., 1973, "Scan of the Cygnus Region in the (1-10) MeV $\gamma$-Ray Energy Range", Astron. Astrophys., 28, 131. [18]

DeGregoria A.J., 1974, "An Investigation of Accretion of Matter onto White Dwarfs as a Possible X-Ray Mechanism", Astrophys. J., 189, 555. [21]

Dessler A.J., 1968, "Solar Wind Interactions and the Magnetosphere", in Physics of the Magnetosphere, ed. R.F. Carovillano, J.F. McClay and H.R. Radoski (Dordrecht: Reidel). [16]

Dilworth C., Maraschi L. and Reina C., 1974, "On the Luminosity Function of Galactic X-Ray Sources", Astron. Astrophys., 28, 71. [23]

Dolan J.F., 1971, "Eclipsing Binary Model of Cygnus XR-1", Nature, 233, 109. [13]

Doroshkevich A.G., Zel'dovich Ya.B. and Novikov D., 1967, "The Origin of Galaxies in an Expanding Universe", Astron. Zh., 44, 295 [Sov. Astron.-AJ, 11, 233]. [12]

Doxey R., Bradt H.V., Levine A., Murthy G.T., Rappaport S. and Spada G., 1973, "XRay Pulse Profile and Celestial Position of Hercules X-1", Astrophys. J. Lett., 182, L25. [16]

Dungey J.W., 1958, Cosmic Electrodynamics (Cambridge: Cambridge University Press). [16]

Eardley D.L. and Lightman A.P., 1975, "Magnetic Viscosity in Relativistic Accretion Disks", Astrophys. J., 200, 187. [26, 27]

Eardley D.L., Lightman A.P. and Shapiro S.L., 1975, "Cygnus X-1: A TwoTemperature Accretion Disk Model which Explains the Observed Hard X-Ray Spectrum", Astrophys. J. Lett., 199, L153. [20, 26]

Eardley D.L., Lightman A.P., Payne D.G. and Shapiro S.L., 1978, "Accretion Disks around Massive Black Holes: Persistent Emission Spectra", Astrophys. J., 224, 53. $[20,27]$

Edelson P.A. and Malkan M.A., 1986, "Spectral Energy Distributions of Active Galactic Nuclei between 0.1 and 100 Microns", Astrophys. J., 308, 59. [1] 
Eichler D., 1979, "Particle Acceleration in Collisionless Shocks: Regulated Injection and High Efficiency", Astrophys. J., 228, 419. [28]

Elsner R.F. and Lamb F.K., 1976, "Accretion Flows in the Magnetospheres of Vela X-1, A0535+26 and Her X-1", Nature, 262, 356. [29]

Elsner R.F. and Lamb F.K., 1977, "Accretion by Magnetic Neutron Stars. I. Magnetospheric Structure and Stability", Astrophys. J., 215, 897. [29]

Elsner R.F., Ghosh P. and Lamb F.K., 1980, "On the Origin and Persistence of LongPeriod Pulsating X-Ray Sources”, Astrophys. J. Lett., 241, L155. [29]

Elvey C.T. and Babcock H.W., 1943, "The Spectra of U Geminorum Type Variable Stars", Astrophys. J., 97, 412. [6]

Elvis M., Czerny B. and Wilkes B. J., 1986, "Continuum Features in Quasars", in The Physics of Accretion onto Compact Objects, ed. K.O. Mason, M.G. Watson and N.E. White (Berlin: Springer). [1]

Evans W.D., Belian R.D., Conner J.P., 1970, "Observations of the Development and Disappearance of the X-Ray Source Centaurus XR-4", Astrophys. J. Lett., 159, L57. [12]

Fabbiano G. and Scherier E.J., 1977, "Further Studies of the Pulsation Period and Orbital Elements of Centaurus X-3", Astrophys. J., 214, 235. [29]

Fabian A.C., 1975, "Slowly Rotating Neutron Stars and Transient X-Ray Sources", Mon. Not. R. astr. Soc., 173, 161. [21, 29]

Fabian A.C., 1980, "The X- and Gamma-Ray Backgrounds", Proc. 10th Texas Symposium on Relativistic Astrophysics ( Ann. NY Acad. Sci., 375, 235). [28]

Fabian A.C., Pringle J.E. and Webbink R.F., 1975, "Ariel 1118-61. A Very Close Binary System or a Slowly Rotating Neutron Star ?", Astroph. Space Sci., 42, 161. [21]

Fabian A.C., Pringle J.E. and Webbink R.F., 1975, "Possible Identification of Ariel 1118-61", Nature, 255, 208. [21]

Fabian A.C., Pringle J.A. and Rees M.J., 1976, "X-Ray Emission from Accretion onto White Dwarfs", Mon. Not. R. astr. Soc., 175, 43. [1]

Feast M.W., 1975, "SY Fornacis and the Mira Ceti B Phenomenon", Observatory, 95, 19. $[21]$

Felten J.E., and Rees M.J., 1972, "Continuum Radiative Transfer in a Hot Plasma with 
Application to Scorpius X-1", Astron. Astrophys., 17, 226. $[13,14,16,18,19$, $26]$

Felten J.E., Rees M.J. and Adams T.F., 1972, "Transfer Effects on X-Ray Lines in Optically Thick Celestial Sources", Astron. Astrophys., 21, 139. [13]

Fishbone L.G. and Moncrief V., 1976, "Relativistic Fluid Disks in Orbit around Kerr Black Holes", Astrophys. J., 207, 962. [25]

Flammang R.A., 1982, "Stationary Spherical Accretion into Black Holes. II. Theory of Optically Thick Accretion", Mon. Not. R. astr. Soc., 199, 833. [1, 28]

Flammang R.A., 1984, "Stationary Spherical Accretion into Black Holes. III. Optically Thick Accretion in Particular Cases", Mon. Not. R. astr. Soc., 206, 589. [1]

Flowers E. and Ruderman M.A., 1977, "Evolution of Pulsar Magnetic Fields", Astrophys. J., 215, 302. [29]

Francey R.J., Fenton A.G., Harries J.R. and McCracken K.G., 1967, "Variability of Centaurus XR-2", Nature, 216, 773. [8]

Frank J., 1978, "Tidal Disruption by a Massive Black Hole and Collisions in Galactic Nuclei", Mon. Not. R. astr. Soc., 184, 87. [27]

Frank J., 1979, "The Fate of the Debris of Tidal Disruption by a Massive Black Hole in a Dense Star Cluster", Mon. Not. R. astr. Soc., 187, 883. [27]

Frank J., King A. R. and Raine D. J., 1986, Accretion Power in Astrophysics (Cambridge: Cambridge University Press). [1]

Frye G:M., Staib J.A., Zych A.D., Hopper W.R., Rawlinson W.R. and Thomas J.A., 1969, "Evidence for a Point Source of High Energy Cosmic Gamma Rays", Nature, 223, 1320. [12]

Fusco-Femiano R. and Massaro E., 1973, "On Gamma-Ray Emission from Cygnus X-2", Astroph. Space Sci., 25, 239. [18]

Galeev A.A., Rosner R. and Vaiana G.S., 1979, "Structured Coronae of Accretion Disks", Astrophys. J., 229, 318. [27]

Ghosh P. and Lamb F.K., 1978, "Disk Accretion by Magnetic Neutron Stars", Astrophys. J. Lett., 223, L83. [29]

Ghosh P. and Lamb F.K., 1979, "Accretion by Rotating Magnetic Neutron Stars. II. Radial and Vertical Structure of the Transition Zone in Disk Accretion", 
Astrophys. J., 232, 259. [29]

Ghosh P., Lamb F.K. and Pethick C.J., 1977, "Accretion by Rotating Magnetic Neutron Stars. I. Flow of Matter inside the Magnetosphere and Its Implications for Spinup and Spin-down of the Star", Astrophys. J., 217, 578. [29]

Giacconi R., Gorenstein P., Gursky H., Husher P.D., Waters J.R., Sandage A., Osmer P. and Peach J.V., 1967, "On the Optical Search for the X-Ray Sources Cyg X-1 and Cyg X-2", Astrophys. J. Lett., 148, L129. [8]

Giacconi R., Gursky H., Kellog E., Levinson R., Scherier E. and Tananbaun H., 1973, "Further X-Ray Observations of Hercules X-1 from UHURU", Astrophys. J., 184, 227. [11]

Giacconi R., Gursky H., Kellog E., Scherier E. and Tananbaun H., 1971, "Discovery of Periodic X-Ray Pulsations in Centaurus X-3 from UHURU", Astrophys. J. Lett., 167, L67. [13]

Ginzburg V.L. and Syrovatskii S.I., 1964, The Origin of Cosmic Rays (New York: MacMillan). [20]

Ginzburg V.L. and Syrovatskii S.I., 1964, "Gamma Rays and Cycloton Radiation XRays of Galactic and Metagalactic Origin", J.E.P.T., 45, 353 [Sov. Astron.$J E P T, 18,245(1963)] .[20]$

Ginzburg V.L. and Syrovatskii S.I., 1965, "Cosmic Magnetobremsstrahlung (Synchrotron Radiation)", Ann. Rev. A. A., 3, 297. [10]

Ginzburg V.L. and Syrovatskii S.I., 1969, Elementary Processes for Cosmic Ray Astrophysics (New York: Gordon and Breach). [20]

Glasby J.S., 1970, The Dwarf Novae (London: Constable \& Co.). [1]

Gnedin Yu.N. and Sunyaev R.A., 1973, "Luminosity of Thermal X-Ray Sources with a Strong Magnetic Field", Mon. Not. R. astr. Soc., 162, 53. [14]

Gorenstein P., Giacconi R. and Gursky H., 1967, "The Spectra of Several X-Ray Sources of Cygnus and Scorpio", Astrophys. J. Lett., 150, L85. [8]

Gould R.J., 1981, "Kinetic Theory of Relativistic Plasma", Phys. Fluids, 24, 102. [28]

Grad H., 1961, "Boundary Layer between a Plasma and a Magnetic Field", Phys. Fluids, 4, 1366. [16] 
Grant G., 1955, "Short-Period Fluctuations in the Intensity of SS Cygni", Astrophys. J., 122, 556. [6]

Grant G. and Abt H.A., 1959, "Photoelectric Photometry of an Outburst of SS Cygni", Astrophys. J., 129, 323. [6]

Greenstein J.L. and Kraft R.P., 1959, "The Binary System Nova DQ Herculis. I. The Spectrum and Radial Velocity during the Eclipse Cycle", Astrophys. J., 130, 99. [6]

Greenstein J.L. and Schmidt M., 1964, "The Quasi-stellar Radio Sources 3C 48 and 3C 273", Astrophys. J., 140, 1. [10]

Greisen K., 1966, "End to the Cosmic-Ray Spectrum ?", Phys. Rev. (Letters), 16, 748. $[20]$

Grindlay J.E., 1978, "Thermal Limit for Spherical Accretion and X-Ray Bursts", Astrophys. J., 221, 234. [28]

Gunn J.E., 1978, Active Galactic Nuclei, ed. C. Hazard and S. Mitton (Cambridge: Cambridge University Press). [27]

Gunn J.E. and Ostriker J.P., 1970, "On the Nature of Pulsars. III. Analysis of Observations", Astrophys. J., 160, 979. [29]

Gusejnov O.Kh. and Zel'dovich Ya.B., 1966, "Collapsed Stars in Binary Systems", Astron. Zh., 43, 313 [Sov. Astron.-AJ, 10, 251]. [12]

Haffner H., 1937, "Ein W Ursae Majoris-Stern in der Praesepe", Z. f. Ap., 14, 285. [6]

Harwitt M. and Salpeter E.E., 1973, "Radiation from Comets near Neutron Stars", Astrophys. J. Lett., 186, L37. [18]

Hasinger G., 1987, "A Classification of Fast Quasi-Periodic X-Ray Oscillators: Is $6 \mathrm{~Hz}$ a Fundamental Frequency?", Astron. Astrophys., 186, 153. [1]

Hatchett S., Buff J. and McCray R., 1976, "Transfer of X-Rays through a Spherically Symmetric Gas Cloud", Astrophys. J., 206, 847. [21]

Hayakawa S., 1974, "Circumstellar Matter in the Accretion Model of Cosmic X-Ray Sources", Prog. Theor. Phys., 50, 459. [21]

Hayakawa S. and Matsuoka M., 1964, "Origin of Cosmic X-Rays", Prog. Theor. Phys. Suppl., 30, 204. [8] 
Haymes R.C. and Harnden F.N.Jr., 1970, "Low-Energy Gamma Radiation from Cygnus", Astrophys. J., 158, 1111. [26]

Haymes R.C., Harnden F.N., Johnson W.N., Prichard H.M. and Bosch H.E., 1972, "The Low-Energy Gamma-Ray Spectrum of Scorpius X-1", Astrophys. J. Lett., 172, L47. $[13,18]$

Herbig G.H., 1944, "The Variable Star UZ Serpentis", Publ. Astron. Soc. Pac., 56, 230. [6]

Herbig G.H., 1960, "Observations and an Interpretation of VV Puppis", Astrophys. J., 132, 76. [6]

Herbst W., Hesser J.E. and Ostriker J.P., 1974, "The 71-Second Variation of DQ Herculis", Astrophys. J., 193, 679. [21]

Herterich K., 1974, "Absorption of Gamma Rays in Intense X-Ray Sources", Nature, 250, 311. [20]

Hills J.G., 1972, "Stellar Debris Clouds in Quasars and Related Objects", Mon. Not. R. astr. Soc., 182, 517. [27]

Hirschfield J.L., Baldwin D.E., and Brown S.C., 1961, "Cyclotron Radiation from a Hot Plasma", Phys. Fluids, 4, 198. [20]

Holloway N., Kundt W. and Wang Y.M., 1978, "Propeller Spindown of Rotating Magnets", Astron. Astrophys., 70, L23. [29]

Holmes A., 1937, The age of the Earth (Nelson Classic). [2]

Holzer T. E. and Axford W. I., 1970, "The Theory of Stellar Winds and Related Flows", Ann. Rev. A. A., 8, 31. [1]

Hoshi R., 1973, "X-Ray Emission from White Dwarfs in Close Binary Systems", Progr. Theor. Phys., 49, 776. [1, 18, 21]

Hoyle F. and Fowler W., 1963, "Nature of Strong Radio Sources", Nature, 197, 533. [5]

Hoyle F. and Lyttleton, 1939, "The Effect of Interstellar Matter in Climatic Variation", Proc. Cam. Phil. Soc., 35, 405. $[1,3,4,12,16]$

Hoyle F. and Lyttleton, 1940, Proc. Camb. Phil. Soc., 36, 325. [3]

Hoyle F. and Lyttleton, 1940, "On the Physical Aspect of Accretion by Stars", Proc. Camb. Phil. Soc., 36, 424. [3] 
Hoyle F. and Narlikar J.V., 1963, "Mach's Principle and the Creation of Matter", Proc. Roy. Soc., 273, No. 1352, 1. [5]

Hoyle F., Fowler W., Burbridge G. and Burbridge M., 1964, "On Relativistic Astrophysics", Astrophys. J., 139, 909. [5, 10]

Huang S.S., 1956, "A Dynamical Problem in Binary Systems and its Bearing on Stellar Evolution", Astron. Astrophys., 61, 49. [6]

Hunt R., 1971, "A Fluid Dynamical Study of the Accretion Processes", Mon. Not. $R$. astr. Soc., 154, 141. $[16,17]$

Ichimaru S., 1978, "Interaction of the Accretion Disk with the Rotating Stellar Magnetic Field: Plasma Diffusion and Angular Momentum Transfer across the Alfvén Surface", Astrophys. J., 224, 198. [29]

Ichimaru S. and Nakano T., 1973, "Plasma Theory of Scorpius X-1", Prog. Theor. Phys., 50, 1867. [21]

Illarionov A.F, and Sunyaev R.A., 1972, "Compton Scattering by Thermal Electrons in X-Ray Sources", Astron. Zh, 49, 58 [Soviet Astr.-AJ, 16, 45, (1972)]. [14, 16, $19,26]$

Illarionov A.F. and Sunyaev R.A., 1975, "Why the Number of Galactic X-Ray Stars is so small ?", Astron. Astrophys., 39, 185. [20, 21, 29]

Inoue H. and Hoshi R., 1975, "X-Ray Emission from a White Dwarf with a Strong Magnetic Dipole Field", Prog. Theor. Phys., 54, 415. [21]

Ipser J. R. and Price R. H., 1977, "Accretion. onto Pregalactic Black Holes", Astrophys. J., 216, 578. [1]

Ipser J. R. and Price R. H., 1982, "Synchrotron Radiation from Spherically Accreting Black Holes", Astrophys. J., 255, 654. [1]

Ipser J. R. and Price R. H., 1983, "Comptonization Effects in Spherical Accretion onto Black Holes", Astrophys. J., 267, 371. [1]

Jackson J.C., 1962, Classical Electrodynamics (New York: Wiley). [20]

Jackson J.C., 1972, "Model of Cygnus X-1", Nature Phys. Sci., 236, 39. [13]

Jaroszynski M., Abramowicz M.A. and Paczynski B., 1980, "Supercritical Accretion Disks around Black Holes", Acta. Astr., 30, 1. [1, 24, 27]

Jeffreys H., 1929, The Earth (Cambridge: Cambridge University Press). [2] 
Jones D.L., Sramek R.A. and Terzian V., 1981, "Extended Radio Emission Aligned with Compact Nuclear Sources in Normal Galaxies", Astrophys. J. Lett., 247, L57. [27]

Johnston M., Bradt H., Doxsey R., Gursky H., Schwartz D. and Schwartz J., 1978, "Position and Pulse Profile of the X-Ray transient 4U 0115+63", Astrophys. J. Lett., 223, L71. [29]

Joss P.C. and Rappaport S.A., 1976, "Observational Constraints on the Masses of Neutron Stars", Nature, 264, 219. [29]

Joss P.C. and Rappaport S.A., 1984, "Neutron Stars in Interacting Binary Systems", Ann. Rev. A. A., 22, 537. [1]

Joy A.H., 1956, "Radial-Velocity Measures of SS Cygni at Minimum Light", Astrophys. J., 124, 317. [6]

Kaplan S.A., 1949, J.E.P.T., 19, 951. [12, 14]

Kaplan S.A., 1954, Doklady Acad. Nauk SSSR, 94, 33. [12]

Kaplan S.A. and Pikel'ner S.B., 1970, The Interstellar Medium (Harvard University Press). [12]

Kaplan S.A. and Tsytovich V.N., 1973, Plasma Astrophysics (Oxford: Pergamon). [20]

Kardashev N.S., 1964, "Magnetic Collapse and the Nature of Intense Sources of Cosmic Radio-frequency Emission", Astron. Zh., 41, 807 [Sov. Astron.-AJ., 8, 643 (1965)]. [12]

Karzas W.J. and Latter R., 1961, "Electron Radiative Transitions in a Coulomb Field", Astrophys. J. Suppl., 6, 167. [15]

Katz J.I., 1975, "The Structure of DQ Herculis", Astrophys. J., 200, 298. [21]

Katz J.I., 1976, "Nonrelativistic Compton Scattering and Models of Quasars", Astrophys. J., 206, 910. [28]

Katz J.I. and Salpeter E.E., 1974, "X-Ray Emission from Vibrating White Dwarfs", Astrophys. J., 193, 429. [21]

Kemp J.C., Swedlund J.B. and Wolstencroft R.D., 1974, "DQ Herculis: Periodic Linear Polarization Synchronous with the Rapid Light Variations", Astrophys. J. Lett., 193, L15. [21] 
Kihara T. and Aono O., 1963, "Unified Theory of Relaxations in Plasmas. I. Basic Theorem", J. Phys. Soc. Japan, 18, 837. [15]

Kitamura M., 1959, Publ. Astr. Soc. Japan, 11, 216. [6]

Klebesadel R.W., Strong I.B. and Olson R.A., 1973, "Observations of Gamma-Ray Bursts of Cosmic Origin", Astrophys. J. Lett., 182, L85. [18]

Klein R. I., Stockman H. S. and Chevalier R. A., 1980, "Supercritical Time-Dependent Accretion onto Compact Objects. I. Neutron Stars", Astrophys. J., 237, 912. [1]

Kolykhalov P.I. and Sunyaev R.A., 1979, "Gamma Emission during Spherically Symmetric Accretion onto Black Holes in Binary Stellar Systems", Astron. Zh., 56, 338 [Soviet Astr. -AJ, 23, 189]. [28]

Kompaneets A.S, 1956, "The Establishment of Thermal Equilibrium between Quanta and Electrons", J.E.P.T., 31, 876 [Sov. Phys.-J.E.P.T., 4, 730 (1957)]. [9, 14, $18,26]$

Koski A.T., 1978, "Spectrophotometry of Seyfert 2 Narrow Galaxies and Narrow-Line Radio Galaxies", Astrophys. J., 223, 56. [27]

Kozlowski M., Jaroszynski M. and Abramowicz M.A., 1978, "The Analytic Theory of Fluid Disks Orbiting the Kerr Black Hole", Astron. Astrophys., 63, 209. [24, 25, 27]

Kozlowski M., Wiita P.J. and Paczynski B., 1979, "Self-Gravitating Accretion Disks Models with Realistic Equations of State and Opacities", Acta Astron., 29, 157. $[25]$

Kraft R.P., 1958, "The Binary System Nova T Coronae Borealis", Astrophys. J., 127, 625. [6]

Kraft R.P., 1959, "The Binary System Nova. DQ Herculis. II. An Interpretation of the Spectrum during the Eclipse Cycle", Astrophys. J., 130, 110. [6]

Kraft R.P., 1962, "Binary Stars among Cataclysmic Variables. I. U Geminorum Stars (Dwarf Novae)", Astrophys. J., 135, 408. [11]

Kraft R.P., 1963, "Cataclysmic Variables as Binary Stars", Adv. Astron. Astroph., 2, 43, ed. Z. Kopal. [11, 14, 21]

Kraft R.P. and Demoulin M.H., 1967, "On the Remarkable Spectroscopic Complexities of Cyg X-2", Astrophys. J. Lett., 150, L183. [8, 12] 
Kraft R.P. and Luyten W.J., 1965, "Binary Stars among Cataclysmic Variables. VI. On the Mean Absolute Magnitude of U Geminorum Variables", Astrophys. J., 142, 1041. [11]

Kristian J., Sandage A.R. and Westphal J., 1967, "Rapid Photometric and Spectroscopic Variations of the X-Ray Source Cyg X-2", Astrophys. J. Lett., 150, L99. [8, 12]

Krzeminski W., 1965, “The Eclipsing Binary U Geminorum”, Astrophys. J., 142, 1051. [11]

Ku W.H.M., Helfand D.J. and Lucy L.B., 1980, "X-Ray Properties of Quasars", Nature, 288, 323. [27]

Kuan P. and Kuhi L.V., 1975, "Cygni Stars and Mass Loss", Astrophys. J., 199, 148. $[21]$

Kuiper J.P., 1941, "On the Interpretation of B Lyrae and Other Close Binaries", Astrophys. J., 93, 133. [6]

Kuiper J.P. and Johnson J.R., 1956, "Dimensions of Contact Surfaces in Close Binaries", Astrophys. J., 123, 90. [6]

Kukarkin B., Parenago P., Efremov Y. and Kholopov P., 1958, General Catalogue of Variable Stars, 1 (Moscow: Akademia Nauk U.S.S.R.). [6]

Kundt W., 1976, "Spinning Neutron Stars and Cosmic Rays", Phys. Letters, 57 A, 195. [29]

Lamb D.Q., 1974, "DQ Herculis: Weak Sister to HZ Herculis", Astrophys. J. Lett., 182, L129. [21]

Lamb F.K., 1977, "Knowledge of Neutron Stars from X-Ray Observations", Proc. 8th Texas Symposium on Relativistic Astrophysics (Ann. NY Acad. Sci., 302, 482). [29]

Lamb D.Q., 1985, "Recent Developments in the Theory of AM Her and DQ Her Stars", in Cataclysmic Variables and Low Mass X-ray Binaries, ed. D.Q. Lamb and J. Patterson. [1]

Lamb D.Q., Lamb F.K. and Pines D., 1973, "Soft Gamma-Ray Bursts from Accreting Compact Objects", Nature Phys. Sci., 246, 52. [18]

Lamb F.K., Pethick C. J. and Pines D., 1973, "A Model for Compact X-Ray Sources: Accretion by Rotating Magnetic Stars", Astrophys. J., 184, 271. [1, 11, 18, 21, 29] 
Lamb F.K., Pines D. and Shaham J., 1978, "Period Variations in Pulsating X-Ray Sources. I. Accretion Flows Parameters and Neutron Star Structure from Timing Observations", Astrophys. J., 224, 969. [29]

Lamb F.K., Pines D. and Shaham J., 1978, "Period Variations in Pulsating X-Ray Sources. II. Torque Variations and Stellar Response", Astrophys. J., 225, 582. $[29]$

Landau L. D. and Lifshitz, 1959, The Classical Theory of Fields (Cambridge, Mass.: Addison-Wesley Press). [8, 24]

Landau L. D. and Lifshitz, 1959, Fluid Mechanics (New York: Pergamon). [1]

Landau L. D. and Lifshitz, 1969, Statistical Physics (Addison-Wesley, Reading). [5]

Lawrence A., 1987, "Classification of Active Galaxies and the Prospect of a Unified Phenomenology", Publ. Astron. Soc. Pac., 99, 309. [1]

Lea S.M., 1976, "Pulsating X-Ray Sources: Slowly Rotating Neutron Stars ?", Astrophys. J. Lett., 208, L69. [29]

Ledoux P. and Walraven Th., 1958, "Variable Stars", Handbuch der Physik, 51 (Berlin: Springer-Verlag). [11]

Levich E.V. and Sunyaev R.A., 1970, "The Heating of Gas in the Vicinity of Quasars, Nuclei of Seyfert Galaxies and Pulsars by the Induced Compton Effects", Astrophys. Lett., 7, 69. [27]

Levich E.V. and Sunyaev R.A., 1971, "Heating of Gas near Quasars, Seyfert-Galaxy Nuclei, and Pulsars by Low-Frequency Radiation", Astron. Zh., 48, 461 [Sov. Astron.-AJ, 15, 363]. [14]

Lewin W.H.G., McClintock J.E., Ryckman S.G. and Smith W.B., 1971, "Detection of a High-Energy X-Ray Flare from a Source in Crux", Astrophys. J. Lett., 166, L69. [13]

Lewin W.H.G., McClintock J.E. and Smith W.B., 1970, "Decrease in the High-Energy X-Ray Flux from Centaurus XR-2", Astrophys. J. Lett., 159, L193. [12]

Liang E.P.T. and Price R.H., 1977, "Accretion Disk Coronae and Cygnus X-1", Astrophys. J., 218, 247. [24]

Liang E.P.T. and Thompson K.A., 1980, "Transonic Disk Accretion onto Black Holes", Astrophys. J., 240, 271. [24] 
Liebert J. and Stockman H. S., 1985, "The AM Herculis Magnetic Variables", in Cataclysmic Variables and Low Mass X-ray Binaries, ed. Patterson J. and Lamb D.Q. (Dordrecht: Reidel). [1]

Lightman A.P., 1974, "Time-dependent Accretion Disks around Compact Objects. I. Theory and Basic Equations", Astrophys. J., 194, 419. [23, 26]

Lightman A.P., 1974, "Time-dependent Accretion Disks around Compact Objects. II. Numerical Models and Instability of Inner Region", Astrophys. J., 194, 429. [1, $23,26]$

Lightman A.P. and Eardley D.M., 1974, "Black Holes in Binary Systems: Instability of Disk Accretion", Astrophys. J. Lett., 187, L1. [1, 11, 22, 25, 26]

Lightman A.P. and Shapiro S.L., 1975, "Spectrum and Polarization of X-Ray from Accretion Disks around Black Holes", Astrophys. J. Lett., 198, L73. [26]

Lightman A.P., Zdziarski A.A. and Rees M.J., 1987, "Effects of Electron-Positron Pair Opacity for Spherical Accretion onto Black Holes", Astrophys. J. Lett., 315, L113. [1]

Linfield R.P., 1981, "VLBI Observations of Jets in Double Radio Galaxies", Astrophys. J., 244, 436. [27]

Lipunov V.M. and Shakura N.I., 1976, "On the Nature of Binary-System X-Ray Pulsars", Soviet Astr. Letters, 2, 133. [29]

Lucy L.B. and Solomon P.M., 1970, "Mass Loss by Hot Stars", Astrophys. J., 158, 879. [21]

Lyndell-Bell D., 1969, "Galactic Nuclei as Collapsed Old Quasars", Nature, 223, 690. $[1,12,13,14,17,25]$

Lyndell-Bell D., 1978, “Gravity Power", Phys. Scripta, 17, 185. [24, 25]

Lyndell-Bell D. and Pringle J.E., 1974, "The Evolution of Viscous Discs and the Origin of the Nebular Variables", Mon. Not. R. astr. Soc., 168, 603. [21, 24, 25]

Lyndell-Bell D. and Rees M.J., 1971, "On Quasars, Dust and the Galactic Center", Mon. Not. R. astr. Soc., 152, 461. [13]

Lynds C.R., 1967, "Spectroscopic Observations of Cyg X-2", Astrophys. J. Lett., 149, L41. [8] 
Lyne A.G., Ritchings R.T. and Smith F.G., 1975, "The Period Derivatives of Pulsars", Mon. Not. R. astr. Soc., 171, 579. [29]

Lyutyi V.M., Sunyaev R.A. and Cherepashchuk A.M., 1972, "Nature of the Optical Variability of HZ Herculis (Her X-1) and BD $+34^{\circ} 3815$ (Cyg X-1)", Astron. Zh., 50, 3 [Sov. Astron.-AJ, 17, 1 (1973)]. [14]

Macdonald D. and Thorne K.S., 1982, "Black-Hole Electrodynamics: an AbsoluteSpace/Universal-Time Formulation", Mon. Not. R. astr. Soc., 198, 345. [27]

Madau P., 1988, “Thick Accretion Disks around Black Holes and the UV/Soft X-Ray Excess in Quasars", Astrophys. J., 327, 116. [1]

Madej J. and Paczynski B., 1977, Nonstationary Evolution of Close Binaries, IInd Symp. Physics and Evolution of Stars, ed. A. Zytkow (Warsaw: Polish Scientific Publisher). [22]

Malkan M.A. and Sargent W.L., 1982, "The Ultraviolet Excess of Seyfert 1 Galaxies and Quasars", Astrophys. J., 254, 22. [1]

Manley O., 1966, "X-Ray Emission from Sco X-1", Astrophys. J., 144, 1253. [8]

Maraschi L., Reina C. and Treves A., 1974, "On Spherical Accretion near Eddington Luminosity", Astrophys. J., 35, 389. [1]

Maraschi L., Reina C. and Treves A., 1978, "The Effect of Radiation Pressure on Spherical Accretion", Astron. Astrophys., 66, 99. [1]

Maraschi L., Roasio R. and Treves A., 1982, "The Effect of Multiple Compton Scattering on the Temperature and Emission Spectra of Accreting Black Holes", Astrophys. J., 253, 312. [1]

Maraschi L., Perola G. C., Reina C. and Treves A., 1979, "Turbolent Accretion onto Massive Black Holes", Astrophys. J., 230, 243. [1]

Margon B. and Ostriker J.P., 1973, "The Luminosity Function of Galactic X-Ray Sources: A Cutoff and a 'Standard Candle' ?", Astrophys. J., 186, 9. [23]

Martynov D.Ya., 1971, Course of General Astrophysics (Moscow: Nauka). [14]

Mason K.O., 1977, "Secular Period Changes in X-Ray Pulsator", Mon. Not. R. astr. Soc., 178, 81P. [29]

Mattews T. and Sandage A., 1962, "3C 196 as a Second Radio Star", Publ. Astron. Soc. Pac., 74, 406. [5] 
Maxon S., 1972, "Bremsstrahlung Rate and Spectra from a Hot Gas $(\mathrm{Z}=1)$ ", Phys. Rev., 5, 1630. $[18,20]$

McCrea W.H., 1953, "The Rate of Accretion of Matter by Stars", Mon. Not. R. astr. Soc., 113, 162. $[4,12]$

McKee C.F., 1970, "Simulation of Counterstreaming Plasmas with Application to Collisionless Electrostatic Shocks", Phys. Rev. (Letters), 24, L990. [18]

Mestel L., 1968, "Magnetic Braking by a Stellar Wind", Mon. Not. R. astr. Soc., 138, 359. [16]

Mestel L., 1971, "Pulsar Magnetosphere", Nature Phys. Sci., 233, 149. [16]

Meszaros P., 1975, "Primeval Black Holes and Galaxy Formation", Astron. Astrophys., 38, 5. $[20]$

Meszaros P., 1975, "Radiation from Spherical Accretion onto Black Holes", Astron. Astrophys., 44, 59. [1, 28]

Meszaros P., 1983, "A Thermal Interpretation of the X-ray Spectra of Quasars, Active Galactic Nuclei and Cygnus X-1", Astrophys. J. Lett., 274, L13. [28]

Meszaros P. and Nagel W., 1985, "X-Ray Pulsar Models. I. Angle-dependent Cyclotron Line Formation and Comptonization", Astrophys. J., 298, 147. [1]

Meszaros P. and Nagel W., 1985, "X-Ray Pulsar Models. II. Comptonized Spectra and Pulse Shape", Astrophys. J., 299, 138. [1]

Meyer F. and Meyer-Hofmeister E., 1981, "On the Elusive Cause of Cataclysmic Variable Outbursts", Astron. Astrophys., 104, L10. [1]

Middleditch J. and Nelson J., 1976, "Studies of Optical Pulsations from HZ Herculis/Hercules X-1: A Determination of the Mass of the Neutron Star", Astrophys. J., 208, 567. [29]

Morrison P., 1967, "Extrasolar X-Ray Sources", Ann. Rev. A. A., 5, 325. [9]

Morton D.C., 1960, "Evolutionary Mass Exchange in Close Binary Systems", Astrophys. J., 132, 146. [6]

Neugebauer G., Oke J.B., Becklin E. and Garmire G., 1969, "A Study of Visual and Infrared Observations of Sco XR-1", Astrophys. J., 155, 1. [13]

Noerdlinger P.D., 1960, "Concerning Certain Collisionless Plasma-Shock Waves Models", Astrophys. J., 133, 1034. [18] 
Norman C.A. and ter Haar D., 1973, "On the Black-Hole Model of Galactic Nuclei", Astron. Astrophys., 24, 121. [17]

Norman C.A. and ter Haar D., 1975, "Plasma Turbolent Reactors: an Astrophysical Paradigm", Phys. Rep., 17, 307. [27]

Novikov I.D. and Thorne K.S., 1973, "Black Hole Astrophysics", in Black Holes, ed. C. DeWitt and B. DeWitt (London and New York: Gordon and Breach). [1, 14, $17,19,23,24,25,26,27]$

Novikov I.D. and Zel'dovich Ya.B., 1966, "Physics of Relativistic Collapse", Suppl. Nuovo Cimento, 4, 810. [9]

Oegelman H., Beuermann K.P., Kanbach G., Mayer-Hasselwander H.A., Capozzi D., Fiordilino E. and Molteni D., 1977, "Increase in the Pulsational Period of 3U0900-40", Astron. Astrophys., 58, 385. [29]

Oppenheimer J. and Snyder H., 1939, "On Continued Gravitational Contraction", Phys. Rev., 56, 455. [5]

Oppenheimer J. and Volkoff G., 1938, "On Massive Neutron Cores", Phys. Rev., 55, 374. [5]

Osaki Y., 1970, "A Mechanism for the Outbursts of U Geminorum Stars", Astrophys. J., 162, 621. [11]

Osaki Y., 1974, "An Accretion Model for the Outbursts of U Geminorum Stars", Publ. Astr. Soc. Japan, 26, 429. [22]

Osterbrock D.E. and Miller J.S., 1975, "The Optical Emission-Line Spectrum of Cygnus A", Astrophys. J., 197, 535. [27]

Ostriker J.P., Rees M.J. and Silk J., 1970, "Some Observable Consequences of Accretion by Defunct Pulsars", Astrophys. Lett., 6, 179. [16]

Ostriker J.P., McCray R., Weaver R. and Yahil A., 1976, "A New Luminosity Limit for Spherical Accretion onto Compact X-Ray Sources", Astrophys. J. Lett., 208, L61. [28]

Pacholczyk A.G., 1970, Radio Astrophysics (San Francisco:Freeman). [20]

Paczynski B., 1965, Acta Astron., 15, 89. [11]

Paczynski B., 1974, "Mass of Cygnus X-1", Astron. Astrophys., 34, 161. [26] 
Paczynski B., 1977, Nonstationary Evolution of Close Binaries, IInd Symposium Physics and Evolution of the Stars, ed. A. Zytkow (Warsaw: Polish Scientific Publishers). [22]

Paczynski B., 1978, "A Model of Selfgravitating Accretion Disk", Acta Astron., 28, 91. $[24,25]$

Paczynski B., 1978, "A Model of Selfgravitating Disk with a Hot Corona", Acta Astron., 28, 241. [25]

Paczynski B. and Sienkiewicz R., 1972, "Evolution of Close Binaries.VIII. Mass Exchange on the Dynamical Time Scale", Acta Astron., 22, 73. [24]

Paczynski B. and Wiita P., 1980, "Thick Accretion Disks and Supercritical Luminosities", Astron. Astrophys., 88, 23. [1, 24, 27]

Pandharipande V.R., Pines D. and Smith R.A., 1976, "Neutron Star Structure: Theory, Observation and Speculation", Astrophys. J., 208, 550. [29]

Papaloizou J.C.B. and Bath G.T., 1975, "Stellar Stability in Close Binary Systems", Mon. Not. R. astr. Soc., 172, 339. [22]

Papaloizou J.C.B. and Pringle J.E., 1977, "Tidal Torques on Accretion Discs in Close Binary Systems", Mon. Not. R. astr. Soc., 181, 441. [22]

Papaloizou J. C. and Pringle J. E., 1984, "The Dynamical Stability of Differentially Rotating Discs with Constant Specific Angular Momentum", Mon. Not. R. astr. Soc., 208, 721. [1]

Papaloizou J. C. and Pringle J. E., 1985, "The Dynamical Stability of Differentially Rotating Discs. II.", Mon. Not. R. astr. Soc., 213, 799. [1]

Papaloizou J., Faulkner J. and Lin D. N., 1983, "On the Evolution of Accretion Disc Flow in Cataclysmic Variables. II. The Existence and Nature of Collective Relaxation Oscillations in Dwarf Nova Systems", Mon. Not. R. astr. Soc., 205, 487. [1]

Parker E. N., 1963, Interplanetary Dynamical Processes (New York: Interscience). [1]

Parker E.N., 1967, "The Dynamical State of the Interstellar Gas and Field. II. Nonlinear Growth of Clouds and Forces in Three Dimensions", Astrophys. J., 149, 517. [10]

Parker E.N., 1972, "Topological Dissipation and the Small-Scale Fields in Turbolent Gases", Astrophys. J., 174, 499. [16] 
Parker E.N. and Ferraro V.C.A., 1971, "Theoretical Aspects of the Worldwide Magnetic Storm Phenomenon", Handbuch der Physik, 49/3 (Berlin: Springer-Verlag). [16]

Pearson T.J., Uwin S.C., Cohen M.H., Linfield R.P., Readhead A.C.S., Seielstad G.A., Simon R.S. and Walker R.C., 1981, "Superluminal Expansion of Quasar 3C 273", Nature, 290, 365. [27]

Peebles P.J.E. and Dicke R.H., 1968, "Origin of the Globular Star Clusters", Astrophys. J., 154, 891. [12]

Petterson J.A., 1978, "On the Occurrence of Streams and Disks in Massive X-Ray Binary Systems", Astrophys. J., 224, 625. [29]

Petterson J.A., Silk J. and Ostriker J.P., 1980, "Variations on a Spherically Symmetrical Accretion Flow", Mon. Not. R. astr. Soc., 191, 571. [28]

Pines D., Pethick C.J. and Lamb F.K., 1972, "Models for Compact X-Ray Sources", Proc, 6th Texas Symposium on Relativistic Astrophysics (Ann. NY Acad. Sci., 224, 237). [11]

Piran T., 1977, "Secondary Winds and Evaporation from Accretion Discs", Mon. Not. R. astr. Soc., 180, 45. [25]

Piran T., 1978, "The Role of Viscosity and Cooling Mechanisms in the Stability of Accretion Disks", Astrophys. J., 221, 652. [1]

Podurets M.A., 1964, "The Collapse of a Star with Back Pressure Taken into Account", Doklady Acad. Nauk SSSR, 154,300 [Sov. Phys.-Doklady, 8, 1]. [5]

Poolley G.G., 1969, "5C3: A Radio Continuum Survey of M31 and its Neighbourhood", Mon. Not. R. astr. Soc., 144, 101. [10]

Pottasch S.R., 1970, "Mass Loss from Stars", in Interstellar Gas Dynamics, I.A.U. Symposium No. 39, (Dordrecht: Reidel). [14]

Pozdnyakov L. A., Sobol' I. M. and Sunyaev R. A., 1983, "Comptonization and the Shaping of X-Ray Source Spectra: Monte Carlo Calculations", Astroph. Space Sci. Rev., 2, 189. [1]

Prendergast K.H., 1960, "The Motion of Gas Streams in Close Binary Systems", Astrophys. J., 132, 162. [14]

Prendergast K.H. and Burbridge G.R., 1968, "On the Nature of Some Galactic X-Ray Sources", Astrophys. J. Lett., 151, L83. [1, 12, 13, 14, 16] 
Pringle J.E., 1975, "Period Changes in Eruptive Binaries", Mon. Not. R. astr. Soc., 170, 633. [21]

Pringle J.E., 1976, "Thermal Instabilities in Accretion Discs", Mon. Not. R. astr. Soc., 177, 65. [27]

Pringle J.E., 1981, "Accretion Discs in Astrophysics", Ann. Rev. A. A., 19, 137. [1, $22,27]$

Pringle J.E. and Rees M.J., 1972, "Accretion Disc Models for Compact X-Ray Sources", Astron. Astrophys., 21, 1. [1, 11, 14, 16, 17, 18, 19, 20, 23, 24, 25, 26, 29]

Pringle J.E., Rees M.J. and Pacholczyk A.G., 1973, "Accretion onto Massive Black Holes", Astron. Astrophys., 29, 179. [1, 19, 26]

Protheroe R.J. and Kazanas D., 1983, "On the Origin of Relativistic Particles and Gamma-Rays in Quasars", Astrophys. J., 265, 620. [28]

Rappaport S. and Joss P.C., 1977, "Accretion Torque in X-Ray Pulsars", Nature, 266, 683. [29]

Rappaport S., Cash W., Doxsey R., McClintock J. and Moore G., 1974, "Possible Detection of Very Soft X-Rays from SS Cygni", Astrophys. J. Lett., 187, L5. $[11,21]$

Rappaport S., Clark G.W., Cominsky L., Joss P.C. and Li F., 1978, "Orbital Elements of $4 \mathrm{U} 0115+63$ and the Nature of the Hard X-Ray Transients", Astrophys. J. Lett., 224, L1. [29]

Readhead A.C.S., Cohen M.H., Pearson T.J. and Wilkinson P.N., 1978, "Bent Beams and the Overall Size of Extragalactic Radio Sources", Nature, 276, 768. [27]

Rees M.J., 1978, "Relativistic Jets and Beam in Radio Galaxies", Nature, 275, 516. $[25]$

Rees M.J., 1978, “Accretion and the Quasar Phenomenon", Phys. Scripta, 17, 193. [1]

Rees M.J., 1984, "Black Hole Models for Active Galactic Nuclei", Ann. Rev. A. A., 22, 471. [1]

Rees M.J., Begelman M.C., Blanford R.D. and Phinney E.S., 1982, "Ion-supported Tori and the Origin of Radio Jets", Nature, 295, 17. [1, 28]

Roberts D.H. and Sturrock P.A., 1973, "Pulsar Magnetospheres, Braking Index, Polar Caps and Period-Pulse Width Distribution", Astrophys. J., 181, 161. [16] 
Robinson E.L., 1973, "High-Speed Photometry of Z Camelopardalis", Astrophys. J., 180, 121. $[11]$

Robinson E.L., 1973, "Detection of Mass Loss from the Dwarf Nova Z Camelopardalis", Astrophys. J., 186, 347. [11]

Robinson E.L., 1976, "The Masses of Cataclysmic Variables", Astrophys. J., 203, 485. $[21]$

Russel H.N., Dugan R.S. and Stewart J.Q., 1938, Astronomy (Ginn \& Co.). [2]

Rybicki G.B. and Lightman A., 1979, Radiative Processes in Astrophysics (New York: Wiley). [1]

Ryle M., 1968, Highlights in Astronomy, ed. L. Perek and D. Reidel. [10]

Sagdeev R.Z., 1966, Rev. Plasma Physics, 4, 23, ed. M.A. Leontovich (New York: Consultants Bureau). [16]

Sahade J., 1959, Liége Symposium: Modeles d'etoiles et evolution stellaire, p. 76. [6]

Salpeter E.E., 1964, "Accretion of Interstellar Matter by Massive Objects", Astrophys. J., 140, 796. [1, 10, 12, 14, 17, 18]

Salpeter E.E., 1973, "Models for Compact X-Ray Sources", in X- and GammaRay Astronomy, I.A.U. Symposium No. 55, ed. H. Bradt and R. Giacconi (Dordrecht: Reidel). [23]

Sandage A., Osmer P., Giacconi R., Gorenstein P., Gursky H., Waters J., Bradt H., Garmire G., Sreekantan B.V., Oda M., Osawa K. and Jugaku J., 1966, "On the Optical Identification of Scorpius X-1", Astrophys. J., 146, 316. [8]

Saslaw W.C., 1968, "The Effects of Accretion on White Dwarf Stars", Mon. Not. R. astr. Soc., 138, 337. [9]

Savonije G.J., 1978, "Roche-lobe Overflow in X-Ray Binaries", Astron. Astrophys., 62, 317. $[29]$

Savonije G.J. and Van den Heuvel E.P.J., 1977, "On the Rotational History of the Pulsars in Massive X-Ray Binaries", Astrophys. J. Lett., 214, L19. [29]

Scharlermann E., 1978, "The Fate of Matter and Angular Momentum in Disk Accretion onto a Magnetized Neutron Star", Astrophys. J., 219, 617. [29]

Schatzman E., 1955, in Gas Dynamics of Cosmic Clouds, I.A.U. Symposium No. 2 (Amsterdam: North Holland Publishing Co.). [4] 
Schreier E., 1977, "Timing Effects in Rotating Neutron Stars", Proc. 8th Symposium on Relativistic Astrophysics (Ann. NY Acad. Sci., 302, 445). [29]

Schreier E., Gursky H., Kellogg E., Tananbaum H. and Giacconi R., 1971, "Further Observations of the Pulsating X-Ray Source Cygnus X-1 from UHURU", Astrophys. J. Lett., 170, L21. [13, 19, 26]

Scherier E., Levinson R., Gursky H., Kellogg E., Tananbaum H. and Giacconi R., 1972, "Evidence for Binary Nature of Centaurus X-3 from UHURU X-Ray Observations", Astrophys. J. Lett., 172, L79. $[13,16]$

Schmidt M., 1962, "Spectrum of a Stellar Object Identified with the Radio Source 3C 286", Astrophys. J., 136, 684. [5]

Schmidt M., 1963, "3C 273: A Star-like Object with Large Red-shift", Nature, 197, 1040. [5]

Schmidt M., 1978, "The Local Space Density of Quasars and Active Nuclei", Phys. Scripta, 17, 135. [27]

Schwarzchild M., 1958, Structure and Evolution of the Stars (Princeton: Princeton University Press). [6]

Shakura N.I., 1972, "Disk Model of Gas Accretion on a Relativistic Star in a Close Binary System", Astron. Zh., 49, 921 [Sov. Astron.-AJ, 16, 756]. [1, 14]

Shakura N.I., 1972, "Effects of Thomson Scattering on the Emission Spectrum of an Optically Semiopaque Plasma", Astron. Zh., 49, $652[$ Sov. Astron.-AJ, 16, 532]. [14]

Shakura N.I., 1972, "The Long Period X-Ray Pulsar 3U0900-40 as a Neutron Star with an Abnormally Strong Magnetic Field", Pis'ma Astron. Zh., 1, 23 [Sov. Astron. Letters, 1, 223). [29]

Shakura N.I. and Sunyaev R.A., 1973, "Black Holes in Binary Systems. Observational Appearance", Astron. Astrophys., 24, 337. [1, 16, 17, 18, 19, 20, 22, 23, 24, 25, $26,27]$

Shakura N.I. and Sunyaev R.A., 1976, "A Theory of the Instability of Disk Accretion on to Black Holes and the Variability of Binary X-Ray Sources, Galactic Nuclei and Quasars", Mon. Not. R. astr. Soc., 175, 613. [1, 25, 27]

Shapiro S.L., 1973, "Accretion onto Black Holes: The Emergent Radiation Spectrum", Astrophys. J., 180, 531. [1, 17, 18, 20, 28] 
Shapiro S.L., 1973, "Accretion onto Black Holes: The Emergent Radiation Spectrum. II. Magnetic Effects", Astrophys. J., 185, 69. [1, 17, 20]

Shapiro S.L., 1974, "Accretion onto Black Holes: The Emergent Radiation Spectrum. III. Rotating (Kerr) Black Holes", Astrophys. J., 189, 343. [26]

Shapiro S.L. and Lightman A.P., 1976, "Black Holes in X-Ray Binaries: Marginal Existence and Rotation Reversals of Accretion Disks", Astrophys. J., 204, 555. $[21,29]$

Shapiro S.L. and Salpeter E.E., 1975, "Accretion onto Neutron Stars under Adiabatic Shock Conditions", Astrophys. J., 198, 671. [1, 21, 26]

Shapiro S.L. and Teukolsky S.A., 1983, Black Holes, White Dwarfs and Neutron Stars (New York N.Y.: Wiley). [1]

Shapiro S.L., Lightman A.P. and Eardley D.M., 1976, "A Two-Temperature Accretion Disk Model for Cygnus X-1: Structure and Spectrum", Astrophys. J, 204, 187. $[1,25,27,28]$

Shields G., 1978, "Thermal Continuum from Accretion Disks in Quasars", Nature, 272, 706. $[1]$

Shkarofsky I.P., Johnston T,W. and Bachynski M.P., 1966, The Particle Kinetics of Plasmas (Reading: Addison Wesley). [15]

Shklovskii I.S., 1962, Astron. Zh., 39, 591 [Sov. Astron.-AJ, 6, 465]. [5]

Shklovskii I.S., 1967, "The Nature of the X-Ray Source Sco X-1", Astron. Zh., 44, 930 [Sov. Astron.-AJ, 11, 749 (1968)]. [9, 12, 14]

Shklovskii I.S., 1967, "On the Nature of the Source of X-Ray Emission of Sco XR-1", Astrophys. J. Lett., 148, L1. [1, 8, 9, 12, 13, 14]

Shonfelder V. and Lichti G., 1974, "Upper Limits to Soft X-Ray Flux from Seven X-Ray Sources and from the Galactic Plane", Astrophys. J. Lett., 192, L1. [18]

Shvartsman V.F., 1970, Radiofisika, 13, 1852. [12]

Shvartsman V.F., 1970, "Ionization Zones around Neutron Stars: $H_{\alpha}$ Emission, Heating of the Interstellar Medium and the Influence on Accretion", Astron. Zh., 47, 824 [Sov. Astron.-AJ, 14, $662(1971)]$. [12]

Shvartsman V.F., 1971, "Halos Around Black Holes", Astron Zh., 48, 479 [Sov. Astron. $A J, 15,377]$. $[1,13,14,16,17,20,26,28]$ 
Shvartsman V.F., 1971, "Neutron Stars in Binary Systems should not be Pulsars", Astron. Zh., 48, 438 [Sov. Astron.-AJ, 15, 342]. [13, 14, 16]

Sikora M., 1981, "Superluminous Accretion Discs", Mon. Not. R. astr. Soc., 196, 257. [27]

Sikora M. and Wilson D.B., 1981, "The Collimation of Particle Beams from Thick Accretion Discs", Mon. Not. R. astr. Soc., 197, 529. [27]

Smak J.I., 1971, "Eruptive Binaries. II. U Geminorum", Acta Astron., 21, 15. [11]

Smak J.I., 1984, "Outbursts of Dwarf Novae", Publ. Astron. Soc. Pac., 96, 5. [1]

Smart W.M., 1938, Stellar Dynamics (Cambridge: Cambridge University Press). [6]

Soltan A., 1982, "Masses of Quasars", Mon. Not. R. astr. Soc., 200, 115. [27]

Sparke L.S. and Shu F.H., 1980, "Extended Radio Doubles are Found in Elliptical Galaxies", Astrophys. J. Lett., 241, L65. [27]

Spitzer L., 1955, Physics of Fully Ionized Gases (New York: Interscience). [10, 15, 16, $18,26,28]$

Spreiter J.R. and Summers A.L., 1967, "On Conditions near the Neutral Points on the Magnetosphere Boundary", Planet. and Space Sci., 15, 787. [16]

Starrfield S.G., 1970, "The Rate of Mass Exchange in DQ Herculis", Astrophys. J., 161, 361. [11]

Starrfield S.G., Truran J.W., Sparks W.M. and Kutter G.S., 1972, "CNO Abundances in Hydrodynamic Models of the Nova Outburst", Astrophys. J., 176, 169. [11]

Strand K.Aa., 1948, "The Parallax of SS Cygni", Astrophys. J., 107, 106. [6]

Strittmatter P.A., Serkowski K., Carswell R., Stein W.A., Merril K.M. and Burbridge E.M., 1972, "Compact Extragalactic. Nonthermal Sources", Astrophys. J. Lett., 175, L7. [17]

Struve O., 1950, Stellar Evolution (Princeton: Princeton University Press). [6, 8]

Sunyaev R.A., 1972, "Variability of X-Rays from Black Holes with Accretion Disks", Astron. Zh., 49, 1153 [Sov. Astron.-AJ, 16, 941 (1973)]. [14, 19]

Sunyaev R.A. and Titarchuk L.G., 1980, "Comptonization of X-Rays in Plasma Cloud. Typical Radiation Spectra", Astron. Astrophys., 86, 121. [1] 
Sunyaev R.A. and Truemper J., 1979, "Hard X-Ray Spectrum of Cyg X-1", Nature, 279, 506. [1]

Swedlund J.B., Kemp J.C. and Wolstencroft R.D., 1975, "Periodic Circular Polarization Synchronous with the Rapid Light Variations", Astrophys. J. Lett., 193, L11. $[21]$

Syrovatskii S.I., 1967, "Direct Transformation of Magnetic-Field Energy into Energy of Fast Particle", in Radio Astronomy and the Galactic System, I.A.U. Symposium No. 31, ed. H. Van Woerden, p. 133 (Academic Press). [10]

Takahara F., Tsuruta S. and Ichimura S., 1981, "X-Rays from Active Galactic Nuclei", Astrophys. J., 251, 26. [1]

Tamazawa S., Toyama K., Kanako N. and Ono Y., 1975, "Optically Thick Accretion onto Black Holes", Astroph. Space Sci., 32, 403. [20]

Tananbaum H., Gursky H., Kellogg E.M., Levinson R., Schreier E. and Giacconi R., 1972, "Discovery of a Periodic Pulsating Binary X-Ray Source in Hercules from UHURU", Astrophys. J. Lett., 174, L143. [16]

Tarter C.B. and Salpeter E.E., 1969, "The Interaction of X-Ray Sources with Optically Thick Environments", Astrophys. J., 156, 953. [21]

Tarter C.B., Tucker W.H. and Salpeter E.E., 1969, "The Interaction of X-Ray Sources with Optically Thin Environments", Astrophys. J., 156, 943. [14, 21]

Tassoul J.L., 1978, Theory of Rotating Stars (Princeton: Princeton University Press). [24]

Taylor G.I., 1937, "Fluid Friction Between Rotating Cylinders. I. Torque Measurements", Proc. Roy. Soc., 157, 546. [14]

Thorne K.S., 1974, "Disk-Accretion onto a Black Hole. II. Evolution of the Hole", Astrophys. J., 191, 507. [27]

Thorne K.S. and Price R.H., 1975, "Cygnus X-1: An Interpretation of the Spectrum and Its Variability", Astrophys. J. Lett., 195, L101. [26]

Thorne K.S. and Zytkow A.N., 1977, "Stars with Degenerate Neutron Cores. I. Structure of Equilibrium Models", Astrophys. J., 212, 832. [28]

Thorne K.S., Flammang R.A. and Zytkow A.N., 1981, "Stationary Spherical Accretion into Black Holes. I. Equations of Structure", Mon. Not. R. astr. Soc., 194, 475. [1] 
Tidman D.A., 1967, "Turbolent Shock Waves in Plasmas", Phys. Fluids, 10, 547. [18]

Treves A., Belloni T., Chiappetti L., Maraschi L., Stella L., Tanzi E.G. and van der Klis M., 1988, "X-Ray Spectrum and Variability of the Black Hole Candidate LMCX-3", Astrophys. J., 325, 119. [1]

Trimble V.L. and Thorne K.S., 1969, "Spectroscopic Binaries and Collapsed Stars", Astrophys. J., 156, 1013. [12]

Trubnikov B.A., 1958, "Plasma Radiation in a Magnetic Field", Soviet Phys.-Doklady, 3, 136. [20]

Trumper J., Pietsch W., Reppin C., Voges W., Staubert R. and Kendziorra E., 1978, "Evidence for Strong Cyclotron Line Emission in the Hard X-Ray Spectrum of Hercules X-1", Astrophys. J. Lett., 218, L105. [29]

Tucker W.H., 1967, "Physical Conditions in Sco X-1", Astrophys. J. Lett., 149, L105. [8]

Turolla R., Nobili L. and Calvani M., 1986, "On Hydrodynamics of Radiatively Driven Winds", Astrophys. J., 303, 573. [1]

van Citters G.W. and Morton D.C., 1970, "Model Atmospheres for B-Type Stars with Blanketing by Ultraviolet Lines", Astrophys. J., 161, 695. [16]

van de Hulst H.C., 1955, Mém. Soc. R. Sci. Liège, 15, 393. [4]

van den Bergh, 1969, "Collapsed Objects in Cluster of Galaxies", Nature, 224, 891. [12] van den Heuvel E.P.J., 1977, "Evolutionary Processes in X-Ray Binaries and their Progenitor Systems", Proc. 8th Texas Symposium on Relativistic Astrophysics (Ann. NY Acad. Sci., 302, 14 ). [29]

Vedenov A.A., Velikhov E.P. and Sagdeev R.Z., 1961, "Stability of Plasma", Usp. Fiz. Nauk, 73, 701 [Sov. Phys.-Usp., 4, 332 (1961)]. [9]

Waggett P.C., Warner P.J. and Baldwin J.E., 1977, "NGC 6251, a Very Large Radio Galaxy with an Exceptional Jet", Mon. Not. R. astr. Soc., 181, 465. [27]

Walker M.F., 1954, "Nova DQ Herculis (1934): an Eclipsing Binary with Very Short Period", Publ. Astron. Soc. Pac., 66, 230. [6]

Walker M.F., 1956, "A Photometric Investigation of the Short-Period Eclipsing Binary, Nova DQ Herculis (1934)", Astrophys. J., 123, 68. [6] 
Walker M.F., 1957, in I.A.U. Symposium, No. 3, ed. G. H. Herbig (Cambridge: Cambridge University Press). [6]

Wallerstein G., 1959, "Three-Color Photometry of U Geminorum during an Outburst", Publ. Astron. Soc. Pac., 71, 316. [6]

Wallerstein G., 1961, "On the Colors of the U Geminorum Variables at Maximum Light", Astrophys. J., 134, 1020. [11]

Warner B., 1972, "Observations of Rapid Blue Variables. III. The Companion to Mira", Mon. Not. R. astr. Soc., 159, 95. [21]

Warner B., 1973, "On the Masses of Cataclysmic Variable Stars", Mon. Not. R. astr. Soc., 162, 189. [11]

Warner B., 1973, "More High-Speed Photometry of Cataclysmic Variables", Sky Telesc., 46, 298. [11]

Warner B. and Nather R.E., 1971, "Observations of Rapid Blue Variables. II. U Geminorum", Mon. Not. R. astr. Soc., 152, 219. [11]

Warner B. and Robinson E.L., 1972, "Non-Radial Pulsations in White Dwarf Stars", Nature Phys. Sci, 239, 2. [11]

Wasiutinski J., 1946, Studies in Hydrodynamics and Structure of Stars and Planets, Oslo. [14]

Webster B.L. and Murdin P., 1972, "Cygnus X-1. A Spectroscopic Binary with a Heavy Companion?", Nature, 235, 37. [13]

Weymann R., 1965, "Diffusion Approximation for a Photon Gas Interacting with a Plasma via the Compton Effect", Phys. Fluids, 8, 2112. [9, 26]

Wheaton W.A., Ulmer M.P., Baity W.A., Datlowe D.W., Elcan M.J., Peterson L.E., Klebesadel R.W., Strong I.B., Cline T.L. and Desai U.D., 1973, "The Direction and Spectra Variability of a Cosmic Gamma-Ray Burst", Astrophys. J. Lett., 185, L57. [18]

White N.E., Mason K.O. and Sanford P.W., 1977, "Evidence for a 581-d Modulation in the Pulse Period of 3 U0352+30", Nature, 267, 229. [29]

White N.E., Stella L. and Parmar A.N., 1988, "The X-Ray Spectral Properties of Accretion Disks in X-Ray Binaries", Astrophys. J., 324, 363. [1] 
White N.E., Mason K.O., Sanford P.W. and Murdin P., 1975, "The X-Ray Behaviour of 3U0352+30 (X Per)", Mon. Not. R. astr. Soc., 176, 201. [29]

Wickramasinghe D.T. and Whelan J.A.J., 1975, "The Periodic Transient X-Ray Sources", Nature, 258, 502. [29]

Wilson D.B. and Rees M.J., 1978, "Induced Compton Scattering in Pulsar Winds", Mon. Not. R. astr. Soc., 185, 297. [27]

Wolfe A.M. and Burbridge G.R., 1970, "Black Holes in Elliptical Galaxies", Astrophys. J., 161, 419. [12]

Woltjer L., 1959, "Emission Nuclei in Galaxies", Astrophys. J., 130, 38. [10]

Wood P.R., 1977, "Mass Transfer Instabilities in Binary Systems", Astrophys. J., 217, 530. $[22]$

Wyckoff S., Wehinger P. and Gehren T., 1981, "The Resolution of Quasar Images", Astrophys. J., 247, 750. [27]

Wyller A.A., 1970, "Observational Aspects of Black Holes in Globular Clusters", Astrophys. J., 160, 443. [12]

Zdziarski A.A., 1985, "Power-Law X-Ray and Gamma-Ray Emission from Relativistic Thermal Plasmas", Astrophys. J., 289, 514. [1]

Zel'dovich Ya.B., 1963, Voprosy Kosmog., 9, 80. [5]

Zel'dovich Ya.B., 1964, "The Fate of a Star and the Evolution of Gravitational Energy upon Accretion", Doklady Acad. Nauk SSSR, 155, 67 [Soviet Phys.-Doklady, 9, $195(1964)] \cdot[1,9,12,14,18]$

Zel'dovich Ya.B. and Gusejnov O.Kh., 1965, "Collapsed Stars in Binaries", Astrophys. J., 144, 840. [12]

Zel'dovich Ya.B. and Novikov I.D., 1964, "Mass of Quasi-Stellar Objects", Doklady Acad. Nauk SSSR, 158, 811 [Sov. Phys.-Doklady, 9, 834 (1965)]. [9]

Zel'dovich Ya.B. and Novikov I.D., 1965, "Relativistic Astrophysics. II.", Usp. Fiz. Nauk, 86, 447 [Sov. Phys-Usp., 8, 522 (1966)]. [9]

Zel'dovich Ya.B. and Novikov I.D., 1971, Relativistic Astrophysics, 1 (Chicago: University Chicago Press). $[1,9,12,13,16,20]$

Zel'dovich Ya.B. and Novikov I.D., 1971, The Theory of the Gravitation and Stars Evolution (Moskow: Nauka). [14] 
Zel'dovich Ya.B. and Raizer Yu.P., 1966, Physics of Shock Waves and High-Temperature Hydrodynamic Phenomena (New York: Academic). [14, 28]

Zel'dovich Ya.B. and Shakura N.I., 1969, "X-Ray Emission Accompanying the Accretion of Gas by a Neutron Star", Astron. Zh. , 46, 225 [Sov. Astron.-AJ, 13, 175]. $[1,12,13,14,15,16,18,26]$

Zel'dovich Ya.B. and Smorodinskii Ya.A., 1961, "On an Upper Limit on the Density of Neutrinos, Gravitons, and Baryons in the Universe", J.E.P.T., 41, 907 [Sov. Phys.-J.E.P.T., 14, 647]. [5]

Ziólkowski J., 1977, "Origin and Present Evolution of Massive X-Ray Binaries", Proc. 8th Texas Symposium on Relativistic Astrophysics (Ann. NY Acad. Sci. , 302, 47 ). [29]

Zuckermann M.C., 1954, "Remarques sur le Phenomene de Nova VII", Ann. d'Ap., 17, 243. [11] 